\title{
A Local Classification of Some Special $(\alpha, \beta)$-Metrics of Constant Flag Curvature
}

\author{
Hongmei Zhu \\ College of Mathematics and Information Science, Henan Normal University, Xinxiang 453007, China \\ Correspondence should be addressed to Hongmei Zhu; zhm403@163.com
}

Received 7 May 2014; Accepted 18 July 2014; Published 17 August 2014

Academic Editor: Giovanni Calvaruso

Copyright (C) 2014 Hongmei Zhu. This is an open access article distributed under the Creative Commons Attribution License, which permits unrestricted use, distribution, and reproduction in any medium, provided the original work is properly cited.

We classify some special Finsler metrics of constant flag curvature on a manifold of dimension $n>2$.

\section{Introduction}

One of the important problems in Finsler geometry is to study and characterize Finsler metrics of constant flag curvature, which is the generalization of sectional curvature in Riemannian geometry. The local structure of Finsler metrics of constant flag curvature has been historically mysterious and their classification seems to be far from being solved.

The $(\alpha, \beta)$-metrics are an important class of Finsler metrics including Randers metrics as the simplest class. By making use of navigation problem, Bao et al. gave a local classification of Randers metrics with constant flag curvature [1]. Recently, Zhou has classified that the $(\alpha, \beta)$-metrics with constant flag curvature in the following form,

$$
F=\frac{(\alpha+\beta)^{2}}{\alpha}
$$

are locally projectively flat [2].

Lately, Shen and Zhao have studied projectively flat $(\alpha, \beta)$ metrics

$$
F=\alpha\left(1+\varepsilon s+2 k s^{2}-\frac{k^{2}}{3} s^{4}\right), \quad s=\frac{\beta}{\alpha},
$$

where $\varepsilon$ is a constant and $k$ is a nonzero constant, and they proved such projectively flat Finsler metrics with constant flag curvature must be locally Minkowskian [3]. Hence, one natural problem is to consider the classification of such metrics with constant flag curvature. In this paper, we prove the following rigidity result.
Theorem 1. Let $F=\alpha+\varepsilon \beta+\left(2 k \beta^{2} / \alpha\right)-\left(k^{2} \beta^{4} / 3 \alpha^{3}\right)$, where the 1 -form $\beta$ is nonzero, $\varepsilon$ is a constant, and $k$ is a nonzero constant, be an $(\alpha, \beta)$-metric on a manifold of dimension $n>2$. Suppose that $F$ is of constant flag curvature; then it must be locally Minkowskian.

\section{Preliminaries}

Let $F$ be a Finsler metric on an $n$-dimensional manifold $M$ and $G^{i}$ the geodesic coefficients of $F$, which are defined by

$$
G^{i}=\frac{1}{4} g^{i l}\left\{\left[F^{2}\right]_{x^{k} y^{l}} y^{k}-\left[F^{2}\right]_{x^{l}}\right\}
$$

For any $x \in M$ and $y \in T_{x} M \backslash\{0\}$, the Riemann curvature $R_{y}=R_{j}^{i}\left(\partial / \partial x^{i}\right) \otimes d x^{j}$ is defined by

$$
R_{j}^{i}=2 \frac{\partial G^{i}}{\partial x^{j}}-\frac{\partial^{2} G^{i}}{\partial x^{k} \partial y^{j}} y^{k}+2 G^{k} \frac{\partial^{2} G^{i}}{\partial y^{k} \partial y^{j}}-\frac{\partial G^{i}}{\partial y^{k}} \frac{\partial G^{k}}{\partial y^{j}} .
$$

$(\alpha, \beta)$-metrics were first introduced by Matsumoto [4]. They are expressed in the following form:

$$
F=\alpha \phi(s), \quad s=\frac{\beta}{\alpha},
$$


where $\alpha=\sqrt{a_{i j}(x) y^{i} y^{j}}$ is a Riemannian metric and $\beta=$ $b_{i}(x) y^{i}$ is a 1 -form. $\phi=\phi(s)$ is a smooth positive function satisfying

$$
\begin{array}{r}
\phi(s)>0, \quad \phi(s)-s \phi^{\prime}(s)+\left(b^{2}-s^{2}\right) \phi^{\prime \prime}(s)>0, \\
\forall|s| \leq b<b_{0} .
\end{array}
$$

For any flag $(P, y)$, where $P=\operatorname{span}\{y, u\} \subset T_{x} M$, the flag curvature is defined by

$$
K(P, y):=\frac{g_{y}\left(R_{y}(u), u\right)}{g_{y}(y, y) g_{y}(u, u)-\left[g_{y}(y, u)\right]^{2}} .
$$

When $F$ is Riemannian, $K(P, y)=K(P)$ is independent of $y \in P$. It is just the sectional curvature of $P$ in Riemannian geometry. $F$ is said to be of scalar curvature if, for any $y \in$ $T_{p} M$, the flag curvature $K(P, y)=K(y)$ is independent of $P$ containing $y \in T_{p} M$ that is equivalent to the following system of equations in a local coordinate system $\left(x^{i}, y^{i}\right)$ in $T M$,

$$
R_{j}^{i}=K F^{2}\left(\delta_{j}^{i}-F^{-1} F_{y^{j}} y^{i}\right) .
$$

If $K$ is a constant, then $F$ is said to be of constant flag curvature.

Let

$$
r_{i j}:=\frac{1}{2}\left(b_{i \mid j}+b_{j \mid i}\right), \quad s_{i j}:=\frac{1}{2}\left(b_{i \mid j}-b_{j \mid i}\right),
$$

where "l" denotes the covariant derivative with respect to the Levi-Civita connection of $\alpha$. Clearly $\beta$ is closed if and only if $s_{i j}=0$. Moreover, we denote

$$
\begin{gathered}
r_{j}^{i}:=a^{i k} r_{k j}, \quad s_{j}^{i}:=a^{i k} s_{k j}, \quad r_{00}:=r_{i j} y^{i} y^{j}, \\
r_{i 0}:=r_{i j} y^{j}, \quad s_{i}:=b_{j} s_{i}^{j}, \quad s_{0}:=s_{i} y^{i}, \\
r:=r_{i j} b^{i} b^{j}, \quad s_{i 0}:=s_{i j} y^{j}, \quad r_{i}:=b^{j} r_{j i}, \\
r^{i}:=a^{i j} r_{j}, \quad s^{i}:=a^{i j} s_{j},
\end{gathered}
$$

where $\left(a^{i j}\right):=\left(a_{i j}\right)^{-1}$ and $b^{i}:=a^{i j} b_{j}$. Let $G^{i}$ and ${ }^{\alpha} G^{i}$ be the geodesic coefficients of $F$ and $\alpha$, respectively. Then we have the following.

Lemma 2 (see [5]). For an $(\alpha, \beta)$-metric $F=\alpha \phi(s), s=(\beta / \alpha)$, the geodesic coefficients $G^{i}$ are given by

$$
G^{i}={ }^{\alpha} G^{i}+\alpha Q s_{0}^{i}+\Theta\left\{r_{00}-2 Q \alpha s_{0}\right\} l^{i}+\Psi\left\{r_{00}-2 Q \alpha s_{0}\right\} b^{i},
$$

where

$$
\begin{aligned}
& Q=\frac{\phi^{\prime}}{\phi-s \phi^{\prime}}, \\
& \Theta=\frac{\phi \phi^{\prime}-s\left(\phi \phi^{\prime \prime}+\phi^{\prime} \phi^{\prime}\right)}{2 \phi\left[\left(\phi-s \phi^{\prime}\right)+\left(B-s^{2}\right) \phi^{\prime \prime}\right]}, \\
& \Psi=\frac{\phi^{\prime \prime}}{2\left[\left(\phi-s \phi^{\prime}\right)+\left(B-s^{2}\right) \phi^{\prime \prime}\right]} .
\end{aligned}
$$

Here $l^{i}:=\left(y^{i} / \alpha\right)$ and $B:=b^{2}$.
Let $u(s, B):=-2 \Theta Q, v(s, B):=-2 \Psi Q$; one has the following.

Proposition 3 (see [6]). For any $(\alpha, \beta)$-metric $F=\alpha \phi(s), s=$ $(\beta / \alpha)$, the Riemannian curvature is given by

$$
R_{j}^{i}={ }^{\alpha} R_{j}^{i}+T_{j}^{i},
$$

where

$$
\begin{aligned}
T_{j}^{i}= & \Delta \delta_{j}^{i}+C_{11} l^{i} l_{j}+C_{12} l^{i} b_{j}+C_{13} l^{i} s_{j}+C_{14} l^{i} r_{j} \\
& +C_{130} l^{i} s_{0 j}+C_{140} l^{i} r_{j 0}-(u \alpha) l^{i} s_{j \mid 0}+(2 u \alpha) l^{i} s_{0 \mid j} \\
& -\left(u Q \alpha^{2}\right) l^{i} s_{k} s_{j}^{k}+(2 \Theta) l^{i} r_{00 \mid j}-(2 \Theta) l^{i} r_{j 0 \mid 0} \\
& -(2 Q \alpha \Theta) l^{i} r_{k 0} s_{j}^{k}+(4 Q \alpha \Theta) l^{i} r_{j k} s_{0}^{k}+C_{21} b^{i} l_{j} \\
& +C_{22} b^{i} b_{j}+C_{23} b^{i} s_{j}+C_{24} b^{i} r_{j}+C_{230} b^{i} s_{0 j}+C_{240} b^{i} r_{j 0} \\
& -(v \alpha) b^{i} s_{j \mid 0}+(2 v \alpha) b^{i} s_{0 \mid j}-\left(v Q \alpha^{2}\right) b^{i} s_{k} s_{j}^{k} \\
& +(2 \Psi) b^{i} r_{00 \mid j}-(2 \Psi) b^{i} r_{j 0 \mid 0}-(2 Q \alpha \Psi) b^{i} r_{k 0} s_{j}^{k} \\
& +(4 Q \alpha \Psi) b^{i} r_{j k} s_{0}^{k}+C_{3} s_{j}^{i} \\
& +\left[C_{31} s^{i} l_{j}+C_{32} s^{i} b_{j}+\left(v Q \alpha^{2}\right) s^{i} s_{j}+(2 Q \alpha \Psi) s^{i} r_{j 0}\right] \\
& +\left[C_{310} s_{0}^{i} l_{j}+C_{320} s_{0}^{i} b_{j}+C_{330} s_{0}^{i} s_{j}\right. \\
& \left.+C_{331} s_{0}^{i} s_{0 j}+C_{340} s_{0}^{i} r_{j 0}\right] \\
& +C_{4} r_{j}^{i}+\left[C_{410} r_{0}^{i} l_{j}+C_{420} r_{0}^{i} b_{j}\right. \\
& +(2 Q \alpha) s_{0 \mid j}^{i}-(Q \alpha) s_{j \mid 0}^{i}+\left[C_{311} s_{0 \mid 0}^{i} l_{j}-Q_{s} s_{0 \mid 0}^{i} b_{j}\right], \\
& \left.-(v \alpha) r_{0}^{i} s_{j}-(2 \Psi) r_{0}^{i} r_{j 0}\right] \\
& \left.C_{332}^{i} s_{0}^{k} l_{j}+C_{333} s_{k}^{i} s_{0}^{k} b_{j}-\left(Q^{2} \alpha^{2}\right) s_{k}^{i} s_{j}^{k}\right] \\
& (20)
\end{aligned}
$$

$$
\begin{aligned}
\Delta= & \frac{r_{00}^{2}}{\alpha^{2}}\left[2 \Theta_{s} \Psi\left(B-s^{2}\right)-\Theta_{s}+\Theta^{2}-2 s \Theta \Psi\right] \\
& +\frac{r_{00} s_{0}}{\alpha}\left[2\left(v \Theta_{s}+u_{s} \Psi\right)\left(B-s^{2}\right)+2 u \Theta\right. \\
& \left.\quad-2 v s \Theta-u_{s}+2 Q \Theta_{s}-2 \Theta_{B}\right] \\
& +\frac{2 r_{00} r_{0}}{\alpha}\left(2 \Theta \Psi-\Theta_{B}\right) \\
& -\frac{r_{00 \mid 0}}{\alpha} \Theta+s_{0}^{2}\left[-2 u_{B}+u^{2}+2 u_{s} v\left(B-s^{2}\right)+2 Q u_{s}\right]
\end{aligned}
$$




$$
\begin{aligned}
& +2 r_{0} s_{0}\left(-u_{B}+2 v \Theta\right)+2 u Q \alpha\left(s_{k} s_{0}^{k}\right)+4 Q \Theta\left(r_{k 0} s_{0}^{k}\right) \quad-s_{0 \mid 0} u_{s}-\frac{r_{00 \mid 0}}{\alpha} \Theta_{s} \text {, } \\
& -u s_{0 \mid 0}, \\
& C_{11}=\frac{r_{00}^{2}}{\alpha^{2}}\left[\left(s \Theta_{s} \Psi_{s}-4 \Theta_{s} \Psi-2 s \Theta_{s s} \Psi\right)\left(B-s^{2}\right)+6 s \Theta \Psi\right. \\
& +\Theta^{2}+6 s^{2} \Theta_{s} \Psi+s \Theta_{s}+s \Theta_{s s}+2 \Theta_{s} \\
& \left.-s^{2} \Theta \Psi_{s}\right] \\
& +\frac{r_{00} s_{0}}{\alpha}\left[\left(-2 s \Psi u_{s s}+s u_{s} \Psi_{s}+s v_{s} \Theta_{s}-2 s v \Theta_{s s}\right.\right. \\
& \left.-5 v \Theta_{s}-2 \Psi u_{s}\right)\left(B-s^{2}\right)+2 s \Theta_{B s}+\Theta u \\
& +s u \Theta_{s}+4 s^{2} \Psi u_{s}-5 Q \Theta_{s}+s u_{s s} \\
& +s \Theta u_{s}+s Q_{s} \Theta_{s}-2 s Q_{s} \Theta_{s s}-s^{2} \Theta v_{s} \\
& \left.+6 s^{2} v \Theta_{s}+7 s v \Theta+u_{s}+2 \Theta_{B}\right] \\
& +\frac{2 r_{00} r_{0}}{\alpha}\left(\Theta_{B}+s \Theta_{B s}+s \Theta \Psi_{s}-2 \Theta \Psi-2 s \Theta_{s} \Psi\right) \\
& +\frac{r_{00 \mid 0}}{\alpha}\left(\Theta+s \Theta_{s}\right) \\
& +s_{0}^{2}\left[\left(s u_{s} v_{s}-3 u_{s} v-2 s u_{s s} v\right)\right. \\
& \times\left(B-s^{2}\right)+4 s^{2} u_{s} v-2 Q u_{s s} s \\
& \left.+s u u_{s}-3 Q u_{s}+2 s u_{B s}+s u_{s} Q_{s}\right] \\
& +2 r_{0} s_{0}\left(-2 s v \Theta_{s}+s \Theta v_{s}+s u_{B s}-3 v \Theta\right) \\
& +\alpha s_{k} s_{0}^{k}\left(s Q_{s} u-Q u-2 s Q u_{s}\right) \\
& +s_{0 \mid 0}\left(s u_{s}\right)+2 r_{k 0} s_{0}^{k}\left(s Q_{s} \Theta-2 s Q \Theta_{s}-3 Q \Theta\right), \\
& C_{12}=\frac{r_{00}^{2}}{\alpha^{2}}\left[\left(2 \Theta_{s s} \Psi-\Theta_{s} \Psi_{s}\right)\left(B-s^{2}\right)\right. \\
& \left.-2 \Theta \Psi-\Theta \Theta_{s}+s \Theta \Psi_{s}-6 s \Theta_{s} \Psi-\Theta_{s s}\right] \\
& +\frac{r_{00} s_{0}}{\alpha}\left[\left(2 v \Theta_{s s}+2 \Psi u_{s s}-u_{s} \Psi_{s}-v_{s} \Theta_{s}\right)\left(B-s^{2}\right)\right. \\
& -\left(Q_{s}+u+6 s v\right) \Theta_{s}+2 Q \Theta_{s s}-2 \Theta_{B s} \\
& \left.-4 s u_{s} \Psi+s v_{s} \Theta-u_{s s}-\Theta u_{s}-2 v \Theta\right] \\
& +\frac{2 r_{00} r_{0}}{\alpha}\left(-\Theta \Psi_{s}+2 \Theta_{s} \Psi-\Theta_{B s}\right) \\
& +s_{0}^{2}\left[\left(2 u_{s s} v-u_{s} v_{s}\right)\left(B-s^{2}\right)-u u_{s}\right. \\
& \left.-Q_{s} u_{s}-2 u_{B s}-4 s u_{s} v+2 Q u_{s s}\right] \\
& -2 r_{0} s_{0}\left(u_{B s}+\Theta v_{s}-2 v \Theta_{s}\right) \\
& +2 r_{k 0} s_{0}^{k}\left(2 Q \Theta_{s}-Q_{s} \Theta\right)+\alpha s_{k} s_{0}^{k}\left(2 Q u_{s}-Q_{s} u\right) \\
& \begin{aligned}
C_{13}=r_{00}[ & \left(2 u_{s} \Psi-\Theta_{s} v\right)\left(B-s^{2}\right)-u \Theta \\
& \left.+4 \Theta_{B}+s v \Theta-u_{s}-Q \Theta_{s}\right] \\
+ & \alpha s_{0}\left[2 u_{B}-u^{2}+Q u_{s}+u_{s} v\left(B-s^{2}\right)\right] \\
- & \alpha r_{0}\left(2 v \Theta+2 u_{B}\right)
\end{aligned} \\
& C_{14}=4 r_{00}\left(\Theta_{B}+\Theta \Psi\right)+4 \alpha s_{0}\left(u_{B}+v \Theta\right) \text {, } \\
& C_{130}=\frac{3 r_{00}}{\alpha}\left[Q \Theta+(1+s Q) \Theta_{s}\right]+3 s_{0}(s Q+1) u_{s} \text {, } \\
& C_{140}=\frac{r_{00}}{\alpha}\left[2 \Theta_{s} \Psi\left(B-s^{2}\right)-2 s \Theta \Psi-\Theta_{s}-2 \Theta^{2}\right] \\
& +s_{0}\left[\left(4 v \Theta_{s}-2 \Psi u_{s}\right)\left(B-s^{2}\right)\right. \\
& \left.+4 Q \Theta_{s}+u_{s}-2 u \Theta-4 \Theta_{B}-4 s v \Theta\right] \\
& -4 r_{0}\left(\Theta_{B}+\Theta \Psi\right) \text {, } \\
& C_{21}=\frac{r_{00}^{2}}{\alpha^{2}}\left[\left(s \Psi_{s}^{2}-2 s \Psi \Psi_{s s}-2 \Psi \Psi_{s}\right)\left(B-s^{2}\right)\right. \\
& \left.+s \Psi_{s s}+\Psi_{s}+4 s^{2} \Psi \Psi_{s}\right] \\
& +\frac{r_{00} s_{0}}{\alpha}\left[\left(2 s \Psi_{s} v_{s}-3 v \Psi_{s}-2 s v \Psi_{s s}-2 s \Psi v_{s s}\right)\left(B-s^{2}\right)\right. \\
& +s v_{s s}+2 s \Psi_{B s}-2 Q s \Psi_{s s}+5 s^{2} v \Psi_{s} \\
& \left.+s Q_{s} \Psi_{s}+2 s^{2} v_{s} \Psi-2 s v \Psi-3 Q \Psi_{s}\right] \\
& +\frac{2 r_{00} r_{0}}{\alpha}\left(-s \Psi \Psi_{s}+s \Psi_{B s}\right) \\
& +s_{0}^{2}\left[\left(s v_{s}^{2}-2 s v v_{s s}-v v_{s}\right)\left(B-s^{2}\right)+3 s^{2} v v_{s}\right. \\
& \left.-2 s Q v_{s s}-3 s v^{2}-Q v_{s}-2 v_{B}+s Q_{s} v_{s}+2 s v_{B s}\right] \\
& +2 s_{0} r_{0}\left(s v_{B s}-\Psi v+s \Psi v_{s}-2 s v \Psi_{s}-v_{B}\right) \\
& +\frac{r_{00 \mid 0}}{\alpha}\left(s \Psi_{s}\right)+2 r_{k 0} s_{0}^{k}\left(-Q \Psi+s \Psi Q_{s}-2 s Q \Psi_{s}\right) \\
& +s_{0 \mid 0}\left(s v_{s}-v\right)+\alpha s_{k} s_{0}^{k}\left(s Q_{s} v+Q v-2 s Q v_{s}\right), \\
& C_{22}=\frac{r_{00}^{2}}{\alpha^{2}}\left[\left(2 \Psi \Psi_{s s}-\Psi_{s}^{2}\right)\left(B-s^{2}\right)-\Psi_{s s}-4 s \Psi \Psi_{s}\right] \\
& +\frac{r_{00} s_{0}}{\alpha}\left[2\left(v \Psi_{s s}+\Psi v_{s s}-\Psi_{s} v_{s}\right)\left(B-s^{2}\right)\right. \\
& -2 \Psi_{B s}-v_{s s}+2 v \Psi \\
& \left.-2 s \Psi v_{s}-5 s v \Psi_{s}+2 Q \Psi_{s s}-Q_{s} \Psi_{s}\right] \\
& +\frac{2 r_{00} r_{0}}{\alpha}\left(-\Psi_{B s}+\Psi \Psi_{s}\right) \\
& +2 s_{0} r_{0}\left(-\Psi v_{s}+2 \Psi_{s} v-v_{B s}\right)
\end{aligned}
$$




$$
\begin{aligned}
& +s_{0}^{2}\left[\left(2 v v_{s s}-v_{s}^{2}\right)\left(B-s^{2}\right)-Q_{s} v_{s}\right. \\
& \left.-2 v_{B s}+2 v^{2}+2 Q v_{s s}-3 s v v_{s}\right] \\
& -\frac{r_{00 \mid 0}}{\alpha} \Psi_{s}-s_{0 \mid 0} v_{s}+\alpha s_{k} s_{0}^{k}\left(2 Q v_{s}-Q_{s} v\right) \\
& +2 r_{k 0} s_{0}^{k}\left(2 Q \Psi_{s}-Q_{s} \Psi\right), \\
& C_{23}=r_{00}\left[\left(-v \Psi_{s}+2 \Psi v_{s}\right)\left(B-s^{2}\right)\right. \\
& \left.+4 \Psi_{B}-Q \Psi_{s}-v_{s}+2 s v \Psi\right] \\
& +\alpha s_{0}\left[v v_{s}\left(B-s^{2}\right)+2 v_{B}+Q v_{s}+s v^{2}\right] \\
& -2 \alpha r_{0}\left(\Psi v+v_{B}\right),
\end{aligned}
$$$$
C_{330}=-\alpha\left[v Q_{s}\left(B-s^{2}\right)+Q Q_{s}+v+s Q v\right],
$$$$
C_{331}=-3 Q^{2}+3 s Q Q_{s}+3 Q_{s} \text {, }
$$$$
C_{340}=-2\left[Q_{s}\left(B-s^{2}\right)+s Q+1\right] \Psi+Q_{s},
$$$$
C_{4}=(2 \Psi) r_{00}+(2 v) \alpha s_{0}
$$$$
C_{410}=\frac{r_{00}}{\alpha} s \Psi_{s}+s_{0}\left(s v_{s}-v\right),
$$$$
C_{420}=-\frac{r_{00}}{\alpha} \Psi_{s}-s_{0} v_{s},
$$$$
C_{332}=\alpha\left(Q-s Q_{s}\right) Q, \quad C_{333}=\alpha Q Q_{s},
$$$$
C_{311}=s Q_{s}-1
$$

Proposition 4 (see [6]). For an $(\alpha, \beta)$-metric $F=\alpha \phi(s), s=$ $(\beta / \alpha)$, the Ricci curvature of $F$ is related to the Ricci curvature ${ }^{\alpha}$ Ric of $\alpha$ by

$$
\text { Ric }={ }^{\alpha} \operatorname{Ric}+T_{m}^{m}
$$

where

$$
\begin{aligned}
& T_{m}^{m}=\frac{r_{00}^{2}}{\alpha^{2}}\left[(n-1) c_{1}+c_{2}\right] \\
& +\frac{1}{\alpha}\left\{r_{00} s_{0}\left[(n-1) c_{3}+c_{4}\right]\right. \\
& +r_{00} r_{0}\left[(n-1) c_{5}+c_{6}\right] \\
& \left.+r_{00 \mid 0}\left[(n-1) c_{7}+c_{8}\right]\right\} \\
& +\left\{s_{0}^{2}\left[(n-1) c_{9}+c_{10}\right]+\left(r r_{00}-r_{0}^{2}\right) c_{11}\right. \\
& +r_{0} s_{0}\left[(n-1) c_{12}+c_{13}\right] \\
& +\left(r_{00} r_{m}^{m}-r_{0 m} r_{0}^{m}+r_{00 \mid m} b^{m}-r_{0 m \mid 0} b^{m}\right) c_{14} \\
& +r_{0 m} s_{0}^{m}\left[(n-1) c_{15}+c_{16}\right] \\
& \left.+s_{0 \mid 0}\left[(n-1) c_{17}+c_{18}\right]+s_{0 m} s_{0}^{m} c_{19}\right\} \\
& +\alpha\left\{r s_{0} c_{20}+s_{m} s_{0}^{m}\left[(n-1) c_{21}+c_{22}\right]\right. \\
& +\left(3 s_{m} r_{0}^{m}-2 s_{0} r_{m}^{m}+2 r_{m} s_{0}^{m}\right. \\
& \left.\left.-2 s_{0 \mid m} b^{m}+s_{m \mid 0} b^{m}\right) c_{23}+s_{0 \mid m}^{m} c_{24}\right\} \\
& +\alpha^{2}\left(s_{m} s^{m} c_{25}+s_{m}^{i} s_{i}^{m} c_{26}\right), \\
& c_{1}=2 \Psi \Theta_{s}\left(B-s^{2}\right)-2 s \Psi \Theta+\Theta^{2}-\Theta_{s}, \\
& c_{2}=\left(2 \Psi \Psi_{s s}-\Psi_{s}^{2}\right)\left(B-s^{2}\right)^{2} \\
& -\left(6 s \Psi \Psi_{s}+\Psi_{s s}\right)\left(B-s^{2}\right)+2 s \Psi_{s},
\end{aligned}
$$




$$
\begin{aligned}
& c_{3}=-4\left(2 Q \Psi \Theta_{s}+Q_{s} \Psi \Theta\right)\left(B-s^{2}\right) \\
& +2\left(Q_{s} \Theta+2 Q \Theta_{s}\right)+4 Q \Theta(s \Psi-\Theta)-2 \Theta_{B}, \\
& c_{4}=\left[-4 \Psi\left(2 Q \Psi_{s s}+Q_{s} \Psi_{s}+Q_{s s} \Psi\right)\right. \\
& \left.+4 Q \Psi_{s}^{2}\right]\left(B-s^{2}\right)^{2} \\
& +\left[-4 \Psi^{2}\left(Q-s Q_{s}\right)+2\left(2 Q_{s s} \Psi+Q_{s} \Psi_{s}+2 Q \Psi_{s s}\right)\right. \\
& \left.-2 \Psi_{s B}+20 s Q \Psi \Psi_{s}\right]\left(B-s^{2}\right) \\
& +2 \Psi\left(Q-s Q_{s}\right)-4 \Psi_{s}-Q_{s s}-10 s Q \Psi_{s}, \\
& c_{5}=4 \Psi \Theta-2 \Theta_{B}, \quad c_{6}=2\left(2 \Psi \Psi_{s}-\Psi_{s B}\right)\left(B-s^{2}\right)-2 \Psi_{s}, \\
& \mathcal{c}_{7}=-\Theta, \quad c_{8}=-\Psi_{s}\left(B-s^{2}\right), \\
& c_{9}=8 Q \Psi\left(Q \Theta_{s}+Q_{s} \Theta\right)\left(B-s^{2}\right) \\
& +4 Q^{2}\left(\Theta^{2}-\Theta_{s}\right)+4 Q\left(\Theta_{B}-\Theta_{s}\right), \\
& c_{10}=\left[4 \Psi^{2}\left(2 Q Q_{s s}-Q_{s}^{2}\right)+8 Q \Psi\left(Q \Psi_{s s}+Q_{s} \Psi_{s}\right)\right. \\
& \left.-4 Q^{2} \Psi_{s}^{2}\right]\left(B-s^{2}\right)^{2} \\
& +\left[-16 s Q \Psi\left(Q \Psi_{s}+Q_{s} \Psi\right)-4 \Psi\left(2 Q Q_{s s}-Q_{s}^{2}\right)\right. \\
& -4 Q\left(Q \Psi_{s s}+Q_{s} \Psi_{s}\right) \\
& \left.+4\left(Q \Psi_{s B}+Q_{s} \Psi_{B}\right)\right]\left(B-s^{2}\right) \\
& -12 s^{2} Q^{2} \Psi^{2}+4(2+3 s Q)\left(Q \Psi_{s}+Q_{s} \Psi\right) \\
& -8 Q^{2} \Psi+2 Q Q_{s s}-Q_{s}^{2}+4 s Q \Psi_{B}+8 B \Psi^{2} Q^{2}, \\
& c_{11}=4 \Psi^{2}+4 \Psi_{B}, \quad c_{12}=4 Q\left(-2 \Psi \Theta+\Theta_{B}\right), \\
& c_{13}=\left[8 \Psi\left(Q_{s} \Psi-Q \Psi_{s}\right)+4\left(Q \Psi_{s B}+Q_{s} \Psi_{B}\right)\right]\left(B-s^{2}\right) \\
& +8 s Q \Psi^{2}+4 Q \Psi_{s}-4(1-s Q) \Psi_{B}, \\
& c_{14}=2 \Psi, \quad c_{15}=4 Q \Theta, \\
& c_{16}=-4\left(Q_{s} \Psi-Q \Psi_{s}\right)\left(B-s^{2}\right)+2 Q_{s}-2(1+2 s Q) \Psi \text {, } \\
& c_{17}=2 Q \Theta, \quad c_{18}=2\left(Q_{s} \Psi+Q \Psi_{s}\right)\left(B-s^{2}\right)-Q_{s}+2 s Q \Psi, \\
& c_{19}=-2 Q^{2}+2(1+s Q) Q_{s}, \quad c_{20}=-8 Q\left(\Psi^{2}+\Psi_{B}\right), \\
& c_{21}=-4 Q^{2} \Theta, \quad c_{22}=-4 Q^{2} \Psi_{s}\left(B-s^{2}\right)+2 Q \Psi, \\
& c_{23}=2 Q \Psi, \quad c_{24}=2 Q, \\
& c_{25}=-4 Q^{2} \Psi, \quad c_{26}=-Q^{2} \text {. }
\end{aligned}
$$

Remark 5. In Proposition 4, we have corrected some terms in the formulas for coefficients $c_{9}$ and $c_{10}$, which are not printed.
Definition 6 (see [7]). Let

$$
D_{j k l}^{i}=\frac{\partial^{3}}{\partial y^{j} \partial y^{k} \partial y^{l}}\left(G^{i}-\frac{1}{n+1} \frac{\partial G^{m}}{\partial y^{m}} y^{i}\right),
$$

where $G^{i}$ are the spray coefficients of $F$. The tensor $D:=$ $D_{j k l}^{i} \partial_{i} \otimes d x^{j} \otimes d x^{k} \otimes d x^{l}$ is called Douglas tensor. A Finsler metric is called Douglas metric if the Douglas tensor vanishes.

Note that an $(\alpha, \beta)$-metric $F=\alpha+\varepsilon \beta+\left(2 k \beta^{2} / \alpha\right)-$ $\left(k^{2} \beta^{4} / 3 \alpha^{3}\right)$ is a Douglas metric if and only if $b_{i \mid j}=\sigma[(1+$ $4 k B) a_{i j}-5 k b_{i} b_{j}$ ] holds for some scalar function $\sigma=\sigma(x)[8]$.

Definition 7 (see [7]). Put

$$
W_{k}^{i}:=A_{k}^{i}-\frac{1}{n+1} \frac{\partial A_{k}^{m}}{\partial y^{m}} y^{i}
$$

where $R=\left(R_{k}^{k} /(n-1)\right), A_{j}^{i}=R_{j}^{i}-R \delta_{j}^{i}$. Then $W=W_{k}^{i}\left(\partial / \partial x^{i}\right) \otimes$ $d x^{k}$ is a tensor on $T M \backslash\{0\}$. it is called the Weyl curvature tensor.

It is Hilbert's fourth problem in the regular case to study and characterize Finsler metrics on an open domain $u c$ $\mathbf{R}^{n}$ whose geodesics are straight lines. Finsler metrics with this property are called projectively flat metrics. A famous theorem of Douglas is in the following.

Theorem 8 (see [9]). A Finsler metric $F$ on a manifold $M$ (dim $M>2$ ) is locally projectively flat if and only if $D=0$ and $W=0$.

\section{Proof of Theorem 1}

In this section, we will prove Theorem 1 . First, we will study the following lemma, because Lemma 4.1 [3] is found to have some wrong. For example, when $k=-\left(1 / 4 b^{2}\right), F$ does not satisfy the definition of $(\alpha, \beta)$-metrics at $s=0$.

Lemma 9. $F=\alpha+\varepsilon \beta+\left(2 k \beta^{2} / \alpha\right)-\left(k^{2} \beta^{4} / 3 \alpha^{3}\right)$ is a Finsler metric if and only if one of the following holds.

(a) If $k>0$, then $b<1 / \sqrt{k}$ and $\varepsilon \in[-\sqrt{k}, \sqrt{k}]$.

(b) If $k<0$, then $b<(1 / 2 \sqrt{-k})$ and $\varepsilon \in[-(23 / 24) \sqrt{-k}$, $(23 / 24) \sqrt{-k}]$, where $b:=\left\|\beta_{x}\right\|_{\alpha}$, for any $x \in M$.

Proof. Let

$$
\phi=1+\varepsilon s+2 k s^{2}-\frac{k^{2} s^{4}}{3}, \quad s=\frac{\beta}{\alpha} .
$$

Direct computations yield

$$
\begin{gathered}
\phi-s \phi^{\prime}=\left(k s^{2}-1\right)^{2}, \\
\phi-s \phi^{\prime}+\left(b^{2}-s^{2}\right) \phi^{\prime \prime}=\left(k s^{2}-1\right)\left(5 k s^{2}-1-4 k b^{2}\right) .
\end{gathered}
$$


From the definition of $(\alpha, \beta)$, we know that, for all $|s| \leq b<b_{0}$, $\phi$ must satisfy

$$
\phi>0, \quad \phi-s \phi^{\prime}+\left(b^{2}-s^{2}\right) \phi^{\prime \prime}>0 .
$$

That is,

$$
\begin{gathered}
1+\varepsilon s+2 k s^{2}-\frac{k^{2} s^{4}}{3}>0 \\
\left(k s^{2}-1\right)\left(5 k s^{2}-1-4 k b^{2}\right)>0 .
\end{gathered}
$$

Particularly, when $s=0$ and $s=b$, it is easy to see that

$$
1+4 k b^{2}>0, \quad k b^{2}-1 \neq 0 .
$$

Now we first discuss the second inequality of (22). It is equivalent to the following two cases.

Let $y_{1}=k s^{2}-1, y_{2}=5 k s^{2}-1-4 k b^{2}$; it is easy to see that $y_{1}$ and $y_{2}$ are quadratic functions with respect to $s$.

Case 1. When

$$
\begin{gathered}
k s^{2}-1>0, \\
5 k s^{2}-1-4 k b^{2}>0,
\end{gathered}
$$

(I) $k>0$.

By the graph of quadratic function and (23), we find that there is no $b$ such that $k s^{2}-1>0$ and $5 k s^{2}-1-4 k b^{2}>0$ always hold for all $|s| \leq b$.

$$
\text { Consider (II) } k<0 \text {. }
$$

By the graph of quadratic function and (23), we conclude the same result with (I).

In any case, Case 1 cannot exist for all $|s| \leq b$.

Case 2. When

$$
\begin{gathered}
k s^{2}-1<0, \\
5 k s^{2}-1-4 k b^{2}<0,
\end{gathered}
$$

(III) $k>0$.

By the graph of quadratic function and (23), the above system of inequality always holds if and only if $b<(1 / \sqrt{k})$.

Consider (IV) $k<0$.

Similarly, the above system of inequality always holds if and only if $b<(1 / 2 \sqrt{-k})$.

In the following, we will discuss the condition satisfying the first inequality of (22). Since

$$
1+\varepsilon s+2 k s^{2}-\frac{k^{2} s^{4}}{3}>0 \Longleftrightarrow\left(k s^{2}-3\right)^{2}-3 \varepsilon s-12<0,
$$

let $y=\left(k s^{2}-3\right)^{2}-3 \varepsilon s-12$.
Consider (1) $k>0$.

In this case, by (III), we know that $|s| \leq b<(1 / \sqrt{k})$. By the graph and monotonicity of quadratic functions, we find

$$
-8-3 \varepsilon s<y \leq-3-3 \varepsilon s
$$

Hence, for all $|s| \leq b<(1 / \sqrt{k}),(26)$ always holds if and only if $1+\varepsilon s>0$. Moreover,

if $\varepsilon>0$, we have $\varepsilon \leq \sqrt{k}$. If $\varepsilon=0,1+\varepsilon s>0$ holds forever for all $|s| \leq b<(1 / \sqrt{k})$. If $\varepsilon<0$, we get $\varepsilon \geq-\sqrt{k}$.

In a word, $\varepsilon \in[-\sqrt{k}, \sqrt{k}]$.

$$
\text { Consider (2) } k<0 \text {. }
$$

In this case, by (IV), we know that $|s| \leq b<(1 / 2 \sqrt{-k})$. By the same way as above, we have

$$
-3-3 \varepsilon s \leq y<-\frac{23}{16}-3 \varepsilon s .
$$

Hence, for all $|s| \leq b<(1 / 2 \sqrt{-k})$, (26) always holds if and only if $(23 / 16)+3 \varepsilon s \geq 0$. Furthermore, if $\varepsilon>0$, we have $\varepsilon \leq(23 / 24) \sqrt{-k}$. If $\varepsilon=0,(23 / 16)+3 \varepsilon s \geq 0$ holds forever for all $|s| \leq b<(1 / \sqrt{k})$. If $\varepsilon<0$, we get $\varepsilon \leq-(23 / 24) \sqrt{-k}$.

In any case, $\varepsilon \in[-(23 / 24) \sqrt{-k},(23 / 24) \sqrt{-k}]$. We complete the proof of the lemma.

Lemma 10. Let $F=\alpha+\varepsilon \beta+\left(2 k \beta^{2} / \alpha\right)-\left(k^{2} \beta^{4} / 3 \alpha^{3}\right)$ be a Finsler metric on a manifold $M^{n}$, where $\varepsilon$ is a constant and $k$ is a nonzero constant. If $F$ is of constant flag curvature, then it must satisfy
(a) $r_{00}=\sigma\left(1+4 k B-5 k s^{2}\right) \alpha^{2}$,
(b) $s_{0}=0$,
(c) $s_{0}^{k} s_{0 k}=0$,

where $\sigma=\sigma(x)$ is a smooth function on $M$.

Proof. By a direct computation, we have

$$
\begin{aligned}
& Q=\frac{3 \varepsilon+12 k s-4 k^{2} s^{3}}{3\left(-1+k s^{2}\right)^{2}}, \\
& \Psi=\frac{2 k}{1+4 k B-5 k s^{2}}, \\
& \Theta=\frac{3 \varepsilon-40 k^{2} s^{3}-15 \varepsilon k s^{2}+8 k^{3} s^{5}}{2\left(1+4 k B-5 k s^{2}\right)\left(3+3 \varepsilon s+6 k s^{2}-k^{2} s^{4}\right)} .
\end{aligned}
$$

Since $F$ is of constant flag curvature, $F$ is also of constant Ricci curvature; that is, the following system of equations holds

$$
{ }^{\alpha} R_{i}^{i}+T_{i}^{i}-K(n-1) F^{2}=0 .
$$


By Proposition 4, we can calculate $T_{i}^{i}$ by Maple

$$
\begin{aligned}
& T_{i}^{i}=\frac{r_{00}^{2}}{\alpha^{2}}\left[(n-1) \frac{\bar{c}_{1}}{A_{1}^{3} A_{3}^{2}}+\frac{\bar{c}_{2}}{A_{1}^{4}}\right] \\
& +\frac{r_{00} s_{0}}{\alpha}\left[(n-1) \frac{\bar{c}_{3}}{A_{1}^{3} A_{2}^{2} A_{3}^{2}}+\frac{\bar{c}_{4}}{A_{1}^{4} A_{2}^{2}}\right] \\
& +\frac{1}{\alpha}\left\{r_{00} r_{0}\left[(n-1) \frac{\bar{c}_{5}}{A_{1}^{2} A_{3}}+\frac{\bar{c}_{6}}{A_{1}^{3}}\right]\right. \\
& \left.+r_{00 \mid 0}\left[(n-1) \frac{\bar{c}_{7}}{A_{1} A_{3}}+\frac{\bar{c}_{8}}{A_{1}^{2}}\right]\right\} \\
& +s_{0}^{2}\left[(n-1) \frac{\bar{c}_{9}}{A_{1}^{3} A_{2}^{4} A_{3}^{2}}+\frac{\bar{c}_{10}}{A_{1}^{4} A_{2}^{5}}\right]-\frac{16 k^{2}}{A_{1}^{2}}\left(r r_{00}-r_{0}^{2}\right) \\
& +r_{0} s_{0}\left[(n-1) \frac{\bar{c}_{12}}{A_{1}^{2} A_{2}^{2} A_{3}}+\frac{\bar{c}_{13}}{A_{1}^{3} A_{2}^{2}}\right] \\
& +\frac{4 k}{A_{1}}\left(r_{00} r_{i}^{i}-r_{0 i} r_{0}^{i}+r_{00 \mid i} b^{i}-r_{0 i \mid 0} b^{i}\right) \\
& +r_{0 i} s_{0}^{i}\left[(n-1) \frac{\bar{c}_{15}}{A_{1} A_{2}^{2} A_{3}}+\frac{\bar{c}_{16}}{A_{1}^{2} A_{2}^{2}}\right] \\
& +s_{0 \mid 0}\left[(n-1) \frac{\bar{c}_{17}}{A_{1} A_{2}^{2} A_{3}}+\frac{\bar{c}_{18}}{A_{1}^{2} A_{2}^{2}}\right]+\frac{\bar{c}_{19}}{A_{2}^{5}} s_{0 i} s_{0}^{i} \\
& +\alpha\left\{r s_{0} \frac{\bar{c}_{20}}{A_{1}^{2} A_{2}^{2}}+s_{i} s_{0}^{i}\left[(n-1) \frac{\bar{c}_{21}}{A_{1} A_{2}^{4} A_{3}}+\frac{\bar{c}_{22}}{A_{1}^{2} A_{2}^{4}}\right]\right\} \\
& +\alpha\left[\left(3 s_{i} r_{0}^{i}-2 s_{0} r_{i}^{i}+2 r_{i} s_{0}^{i}-2 s_{0 \mid i} b^{i}+s_{i \mid 0} b^{i}\right)\right. \\
& \left.\times \frac{\bar{c}_{23}}{A_{1} A_{2}^{2}}+\frac{\bar{c}_{24}}{A_{2}^{2}} s_{0 \mid i}^{i}\right]+\alpha^{2}\left(\frac{\bar{c}_{25}}{A_{1} A_{2}^{4}} s_{i} s^{i}+\frac{\bar{c}_{26}}{A_{2}^{4}} s_{i}^{j} s_{j}^{i}\right),
\end{aligned}
$$

where

$$
\begin{aligned}
& A_{1}=1+4 k B-5 k s^{2}, \quad A_{2}=-1+k s^{2}, \\
& A_{3}=3+3 \varepsilon s+6 k s^{2}-k^{2} s^{4}
\end{aligned}
$$

and $\bar{c}_{i}(i=1,2, \ldots, 26)$ are polynomials of $s$.

Plugging (31) into (30) and multiplying it by $A_{1}^{4}$ yield

$$
\begin{gathered}
{ }^{\alpha} R_{i}^{i}\left(1+4 k B-5 k s^{2}\right)^{4}+T_{i}^{i}\left(1+4 k B-5 k s^{2}\right)^{4} \\
-K(n-1) F^{2}\left(1+4 k B-5 k s^{2}\right)^{4}=0 .
\end{gathered}
$$

It is easy to see that

$$
\begin{aligned}
& { }^{\alpha} R_{i}^{i}\left(1+4 k B-5 k s^{2}\right)^{4}-K(n-1) F^{2}\left(1+4 k B-5 k s^{2}\right)^{4} \\
& \quad \equiv 0 \bmod \left(1+4 k B-5 k s^{2}\right)
\end{aligned}
$$

Hence,

$$
T_{i}^{i}\left(1+4 k B-5 k s^{2}\right)^{4} \equiv 0 \bmod \left(1+4 k B-5 k s^{2}\right) .
$$

From (31) we find that

$$
\frac{r_{00}^{2}}{\alpha^{2}} \bar{c}_{2}+\frac{r_{00} s_{0}}{\alpha} \frac{\bar{c}_{4}}{A_{2}^{2}}+\frac{\bar{c}_{10}}{A_{2}^{5}} s_{0}^{2} \equiv 0 \bmod \left(1+4 k B-5 k s^{2}\right) .
$$

By Lemma 9, we need to divide two cases.

Consider (1) $k>0$.

In this case, we know that $k \in(0,(1 / B))$, where $B:=b^{2}=$ $\|\beta\|_{\alpha}^{2}$. Hence, $((1+4 k B) / 5 k)>0$.

For

$$
\begin{aligned}
1+4 k B-5 k s^{2} & =-5 k\left(s^{2}-\frac{1+4 k B}{5 k}\right) \\
& =-5 k\left(s-\sqrt{\frac{1+4 k B}{5 k}}\right)\left(s+\sqrt{\frac{1+4 k B}{5 k}}\right),
\end{aligned}
$$

we get

$$
\begin{aligned}
& \frac{r_{00}^{2}}{\alpha^{2}} \bar{c}_{2}+\frac{r_{00} s_{0}}{\alpha} \frac{\bar{c}_{4}}{A_{2}^{2}}+\frac{\bar{c}_{10}}{A_{2}^{5}} s_{0}^{2} \equiv 0 \bmod \left(s+\sqrt{\frac{1+4 k B}{5 k}}\right), \\
& \frac{r_{00}^{2}}{\alpha^{2}} \bar{c}_{2}+\frac{r_{00} s_{0}}{\alpha} \frac{\bar{c}_{4}}{A_{2}^{2}}+\frac{\bar{c}_{10}}{A_{2}^{5}} s_{0}^{2} \equiv 0 \bmod \left(s-\sqrt{\frac{1+4 k B}{5 k}}\right) .
\end{aligned}
$$

Simplifying (38) by Maple yields

$$
\begin{aligned}
& \left(\frac{r_{00}}{\alpha}-\frac{8 k \sqrt{(5(1+4 k B) / k)}(2 k B-7)+75 \varepsilon}{24(k B-1)^{2}} s_{0}\right)^{2} \\
& \quad \equiv 0 \bmod \left(s+\sqrt{\frac{1+4 k B}{5 k}}\right), \\
& \left(\frac{r_{00}}{\alpha}+\frac{8 k \sqrt{(5(1+4 k B) / k)}(2 k B-7)-75 \varepsilon}{24(k B-1)^{2}} s_{0}\right)^{2} \\
& \quad \equiv 0 \bmod \left(s-\sqrt{\frac{1+4 k B}{5 k}}\right) .
\end{aligned}
$$

Since $k \in(0,(1 / B)), 2 k B-7 \neq 0$. Moreover, we note that $\varepsilon \neq$ $\pm(8 k / 75) \sqrt{(5(1+4 k B) / k)}(2 k B-7)$. Otherwise, by Lemma 9 , $F$ is not a Finsler metric. 
From (39), and Zhou's Lemma 4.1 [2], we obtain that

$$
\begin{gathered}
r_{00}+\frac{\sqrt{5 k}[8 k \sqrt{(5(1+4 k B) / k)}(2 k B-7)+75 \varepsilon]}{24(k B-1)^{2} \sqrt{1+4 k B}} \alpha s s_{0} \\
=\sigma_{1}\left(1+4 k B-5 k s^{2}\right) \alpha^{2}, \\
r_{00}+\frac{\sqrt{5 k}[8 k \sqrt{(5(1+4 k B) / k)}(2 k B-7)-75 \varepsilon]}{24(k B-1)^{2} \sqrt{1+4 k B}} \alpha s s_{0} \\
=\sigma_{2}\left(1+4 k B-5 k s^{2}\right) \alpha^{2},
\end{gathered}
$$

where $\sigma_{1}=\sigma_{1}(x)$ and $\sigma_{2}=\sigma_{2}(x)$ are smooth functions on a manifold $M^{n}$.

It is easy to see that

$$
s s_{0} \equiv 0 \bmod \left(1+4 k B-5 k s^{2}\right) .
$$

The above equation holds if and only if

$$
s_{0}=0 \text {. }
$$

Therefore, we have

$$
r_{00}=\sigma\left(1+4 k B-5 k s^{2}\right) \alpha^{2},
$$

where $\sigma=\sigma(x)$ is a smooth function on a manifold $M^{n}$.

Consider (2) $k<0$.

In this case, we know that $k \in(-(1 / 4 B), 0)$, where $B:=$ $b^{2}=\|\beta\|_{\alpha}^{2}$. Hence, $((1+4 k B) / 5 k)<0$. Moreover, $1+4 k B-5 k s^{2}$ is irreducible with respect to $s$.

Simplifying (36) directly by Maple yields

$$
\begin{aligned}
& {\left[\frac{r_{00}}{\alpha}+\frac{8(2 k B-7)\left(5 k s+\sqrt{5 k\left(5 k s^{2}-4 k B-1\right)}\right)-75 \varepsilon}{24(1-k B)^{2}} s_{0}\right]} \\
& \times\left[\frac{r_{00}}{\alpha}\right. \\
& \left.+\frac{8(2 k B-7)\left(5 k s-\sqrt{5 k\left(5 k s^{2}-4 k B-1\right)}\right)-75 \varepsilon}{24(1-k B)^{2}} s_{0}\right] \\
& \equiv 0 \bmod \left(1+4 k B-5 k s^{2}\right) \text {. }
\end{aligned}
$$

By Lemma 9, we note that $5 k s^{2}-4 k B-1<0$. The above equation holds if and only if

$$
\begin{aligned}
r_{00} & +\frac{8(2 k B-7)\left(5 k s+\sqrt{5 k\left(5 k s^{2}-4 k B-1\right)}\right)-75 \varepsilon}{24(1-k B)^{2}} \alpha s_{0} \\
& =\sigma_{3}\left(1+4 k B-5 k s^{2}\right) \alpha^{2}
\end{aligned}
$$

or

$$
\begin{aligned}
r_{00} & +\frac{8(2 k B-7)\left(5 k s-\sqrt{5 k\left(5 k s^{2}-4 k B-1\right)}\right)-75 \varepsilon}{24(1-k B)^{2}} \alpha s_{0} \\
& =\sigma_{4}\left(1+4 k B-5 k s^{2}\right) \alpha^{2}
\end{aligned}
$$

where $\sigma_{3}=\sigma_{3}(x)$ and $\sigma_{4}=\sigma_{4}(x)$ are smooth functions on a manifold $M^{n}$.

In any case, it is easy to find that $s_{0}=0$. Hence, $r_{00}=$ $\sigma\left(1+4 k B-5 k s^{2}\right) \alpha^{2}$, where $\sigma=\sigma(x)$ is a smooth function on a manifold $M^{n}$.

Plugging (31) and $s_{0}=0$ into (30) and multiplying it by $A_{2}^{5}$, we have

$$
s_{0 i} s_{0}^{i} \bar{c}_{19} \equiv 0 \bmod \left(-1+k s^{2}\right)
$$

where

$$
\begin{aligned}
\bar{c}_{19}=-\frac{2}{9}( & 20 k^{5} s^{8}-160 k^{4} s^{6}-48 \varepsilon k^{3} s^{5}+360 k^{3} s^{4} \\
& \left.+240 \varepsilon k^{2} s^{3}+45 \varepsilon^{2} k s^{2}+36 k-9 \varepsilon^{2}\right) .
\end{aligned}
$$

Simplifying (47) by Maple, we have

$$
s_{0 i} s_{0}^{i}\left(192 \varepsilon k s+256 k+36 \varepsilon^{2}\right) \equiv 0 \bmod \left(-1+k s^{2}\right) .
$$

So

$$
s_{0 i} s_{0}^{i}=0
$$

Lemma 11. Let $F=\alpha+\varepsilon \beta+\left(2 k \beta^{2} / \alpha\right)-\left(k^{2} \beta^{4} / 3 \alpha^{3}\right)$ be a Finsler metric on an $n$-dimensional manifold $M$, where $\varepsilon$ is a constant and $k$ is a nonzero constant. Suppose that $F$ is of constant flag curvature; then $\beta$ is closed and $K=0$.

Proof. By Lemma 10, we have

$$
\begin{gathered}
r_{i j}=\sigma\left[(1+4 k B) a_{i j}-5 k b_{i} b_{j}\right], \\
s_{i}=0, \quad s_{k}^{i} s_{j}^{k}=0,
\end{gathered}
$$

where $\sigma=\sigma(x)$ is a smooth function on an $n$-dimensional manifold $M$.

Thus, by some computations, we have

$$
\begin{gathered}
r_{0}=\sigma \beta(1-k B), \quad r=\sigma(1-k B) B, \\
r_{00 \mid 0}=\sigma_{0} \alpha^{2}\left(1+4 k B-5 k s^{2}\right)+2 k \sigma \alpha\left(4 \alpha r_{0}-5 s r_{00}\right), \\
r_{00 \mid j} b^{j}=\sigma_{b} \alpha^{2}\left(1+4 k B-5 k s^{2}\right)+2 k \sigma \alpha\left(4 \alpha r-5 s r_{0}\right),
\end{gathered}
$$




$$
\begin{gathered}
r_{j 0 \mid 0} b^{j}=\sigma_{0}(1-k B) \beta+k \sigma\left(3 \beta r_{0}-5 B r_{00}\right), \\
r_{i}^{i}=\sigma[(1+4 k B) n-5 k B], \\
r_{i 0} r_{0}^{i}=\sigma^{2} \alpha^{2}\left[(1+4 k B)^{2}-5 k s^{2}(2+3 k B)\right], \\
r_{j 0}=\sigma \alpha\left[(1+4 k B) l_{j}-5 k s b_{j}\right], \quad r_{j}=\sigma(1-k B) b_{j}, \\
r_{j 0 \mid 0}=\sigma_{0} \alpha\left[(1+4 k B) l_{j}-5 k s b_{j}\right] \\
+\sigma k \alpha\left(8 r_{0} l_{j}-5 \frac{r_{00}}{\alpha} b_{j}-5 s r_{j 0}+5 s s_{0 j}\right), \\
r_{00 \mid j}=\sigma_{j} \alpha^{2}\left(1+4 k B-5 k s^{2}\right) \\
+2 \sigma k \alpha\left(4 r_{j} \alpha-5 s r_{j 0}-5 s s_{0 j}\right), \\
r_{0}^{i}=\sigma \alpha\left[(1+4 k B) l^{i}-5 k s b^{i}\right], \\
r_{j}^{i}=\sigma\left[(1+4 k B) \delta_{j}^{i}-5 k b^{i} b_{j}\right],
\end{gathered}
$$

where $\sigma_{0}:=\sigma_{x^{i}} y^{i}, \sigma_{b}:=\sigma_{x^{i}} b^{i}, r_{0}:=r_{i} y^{i}, l_{j}:=\left(y_{j} / \alpha\right), l^{i}:=$ $a^{i j} l_{j}=\left(y^{i} / \alpha\right),\left(a^{i j}\right):=\left(a_{i j}\right)^{-1}$.

Case $1(\varepsilon \neq 0)$. Plugging the above equations into the expression of $T_{j}^{i}$ in Proposition 3 and simplifying it by Maple, we have

$$
\begin{aligned}
& T_{j}^{i}=\left(\frac{T_{1}}{A_{3}} \sigma^{2}+T_{2} \sigma_{0}\right) \frac{\alpha^{2}}{A_{3}} \delta_{j}^{i} \\
& +\left[\frac{T_{3}}{A_{3}^{2}} \alpha \sigma^{2}+\left(\frac{T_{4}}{A_{3}} \alpha+T_{5}\right) \frac{\sigma_{0}}{A_{1}}\right] \frac{\alpha}{A_{3}} l^{i} l_{j} \\
& +\left[\frac{T_{6}}{A_{3}^{2}} \alpha \sigma^{2}+\left(\frac{T_{7}}{A_{3}} \alpha+T_{8}\right) \frac{\sigma_{0}}{A_{1}}\right] \frac{\alpha}{A_{3}} l^{i} b_{j} \\
& +\frac{\alpha}{A_{2}^{2} A_{3}}\left(T_{9} \sigma+T_{10}\right) l^{i} s_{0 j}+\frac{T_{11}}{A_{3}} \alpha^{2} l^{i} \sigma_{j} \\
& +4 \alpha k\left(\frac{5 \alpha k s^{2}-4 k B-1}{A_{1}} \sigma_{0}+\alpha k s \sigma^{2}\right) b^{i} l_{j} \\
& +4 \alpha k^{2}\left[\frac{5 s(1-\alpha)}{A_{1}} \sigma_{0}-\alpha \sigma^{2}\right] b^{i} b_{j} \\
& +\frac{\alpha \sigma T_{12}}{A_{2}^{2}} b^{i} s_{0 j}+4 k \alpha^{2} b^{i} \sigma_{j}-\frac{20 k^{2} s A_{3}}{A_{2}^{3}}\left(s l_{j}-b_{j}\right) s_{0}^{i} \\
& +\frac{T_{13}}{A_{2}^{5}} s_{0}^{i} s_{0 j}+\frac{T_{14} \alpha}{A_{2}^{2}}\left(2 s_{0 \mid j}^{i}-s_{j \mid 0}^{i}\right) \\
& +\frac{T_{15}}{A_{2}^{3}} s_{0 \mid 0}^{i} l_{j}+\frac{T_{16}}{A_{2}^{3}} s_{0 \mid 0}^{i} b_{j},
\end{aligned}
$$

where $T_{i}(i=1, \ldots, 16)$ are polynomials of $s, A_{1}=1+4 k B-$ $5 k s^{2}, A_{2}=-1+k s^{2}$, and $A_{3}=3+3 \varepsilon s+6 k s^{2}-k^{2} s^{4}$.
Because $F$ has constant flag curvature, it is equivalent to

$$
{ }^{\alpha} R_{j}^{i}+T_{j}^{i}=K F^{2}\left(\delta_{j}^{i}-\frac{y^{i}}{F} F_{y^{j}}\right) .
$$

Substitute (53) into (54) and multiplying it by $A_{2}^{5}$, we find

$$
s_{0}^{i} s_{0 j} T_{13} \equiv 0 \bmod \left(k s^{2}-1\right),
$$

where $T_{14}=-(1 / 3)\left(20 k^{5} s^{8}-160 k^{4} s^{6}-48 \varepsilon k^{3} s^{5}+360 k^{3} s^{4}+\right.$ $\left.240 \varepsilon k^{2} s^{3}+45 \varepsilon^{2} k s^{2}+36 k-9 \varepsilon^{2}\right)$.

By the same way as Lemma 10, we conclude $s_{0}^{i}=0$ or $s_{0 j}=0$; that is, $\beta$ is closed.

Case $2(\varepsilon=0)$. By the same approach as the above, we still conclude that $\beta$ is closed.

Now we will prove $K=0$.

Let $\phi=1+\varepsilon s+2 k s^{2}-\left(k^{2} s^{4} / 3\right)$. If $F=\alpha \phi(s), s=$ $\beta / \alpha$, has constant flag curvature $K$, then $F$ has constant Ricci curvature. That means that Ric $=(n-1) K F^{2}$. Hence, $F$ is an Einstein metric with Ricci constant $K$.

Note that $\phi=1+\varepsilon s+2 k s^{2}-\left(k^{2} s^{4} / 3\right)$ is a polynomial in $s$ of degree 4. According to Theorem 1.1 (see [6]), we know that it is Ricci flat. So $K=0$.

Proof of Theorem 1. By Lemma 10, we have

$$
r_{i j}=\sigma\left[(1+4 k B) a_{i j}-5 k b_{i} b_{j}\right],
$$

where $\sigma=\sigma(x)$ is a smooth function on an $n$-dimensional manifold.

Moreover, by Lemma 11, we know that $\beta$ is closed; that is, $s_{i j}=0$, Hence,

$$
b_{i \mid j}=\sigma\left[(1+4 k B) a_{i j}-5 k b_{i} b_{j}\right] .
$$

(1) If $\sigma=0$, then $b_{i \mid j}=0$; that is, $\beta$ is parallel with respect to $\alpha$. By the proof of Lemma 11, we find that the sectional curvature of $\alpha$ equals 0 . That means that $\alpha$ is flat. Therefore, $F$ is locally Minkowskian.

(2) If $\sigma \neq 0$.

By (57), we obtain that $F$ is a Douglas metric. That means that the Douglas curvature tensor $D=0$.

Since $F$ has constant flag curvature, the Weyl curvature tensor $W=0$. By Theorem 8 , we know that $F$ must be locally projectively flat. Hence, by Theorem 1.3 (see [3]), $F$ is locally Minkowskian.

\section{Conflict of Interests}

The author declares that there is no conflict of interests regarding the publication of this paper.

\section{Acknowledgment}

The author is grateful to the referee for the useful comments. 


\section{References}

[1] D. Bao, C. Robles, and Z. Shen, "Zermelo navigation on Riemannian manifolds," Journal of Differential Geometry, vol. 66, no. 3, pp. 377-435, 2004.

[2] L. Zhou, "A local classification of a class of $(\alpha, \beta)$ metrics with constant flag curvature," Differential Geometry and Its Applications, vol. 28, no. 2, pp. 170-193, 2010.

[3] Y. Shen and L. Zhao, "Some projectively flat $(\alpha, \beta)$-metrics," Science in China. Series A, vol. 49, no. 6, pp. 838-851, 2006.

[4] M. Matsumoto, "The Berwald connection of Finsler space with an $(\alpha, \beta)$-metric," Tensor, vol. 50, pp. 18-21, 1991.

[5] S. S. Chern and Z. Shen, Riemann-Finsler Geometry, vol. 6 of Nankai Tracts in Mathematics, World Scientific, Hackensack, NJ, USA, 2005.

[6] X. Cheng, Z. Shen, and Y. Tian, "A class of Einstein $(\alpha, \beta)$ metrics," Israel Journal of Mathematics, vol. 192, no. 1, pp. 221249, 2012.

[7] Z. Shen, Diffierential Geometry of Spray and Finsler Spaces, Kluwer Academic, 2001.

[8] B. Li, Y. Shen, and Z. Shen, "On a class of Douglas metrics," Studia Scientiarum Mathematicarum Hungarica: A Quarterly of the Hungarian Academy of Sciences, vol. 46, no. 3, pp. 355-365, 2009.

[9] J. Douglas, “The general geometry of paths," Annals of Mathematics, vol. 29, no. 1-4, pp. 143-168, 1927/28. 


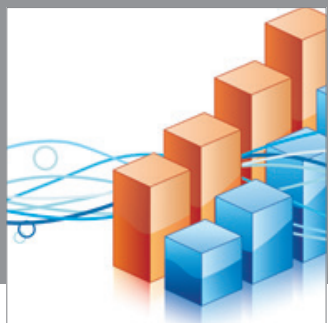

Advances in

Operations Research

mansans

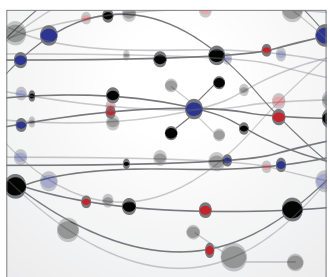

The Scientific World Journal
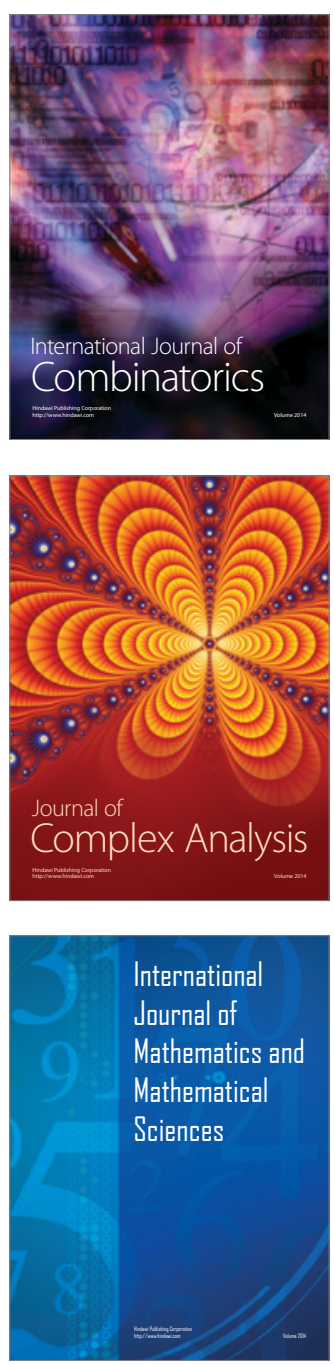
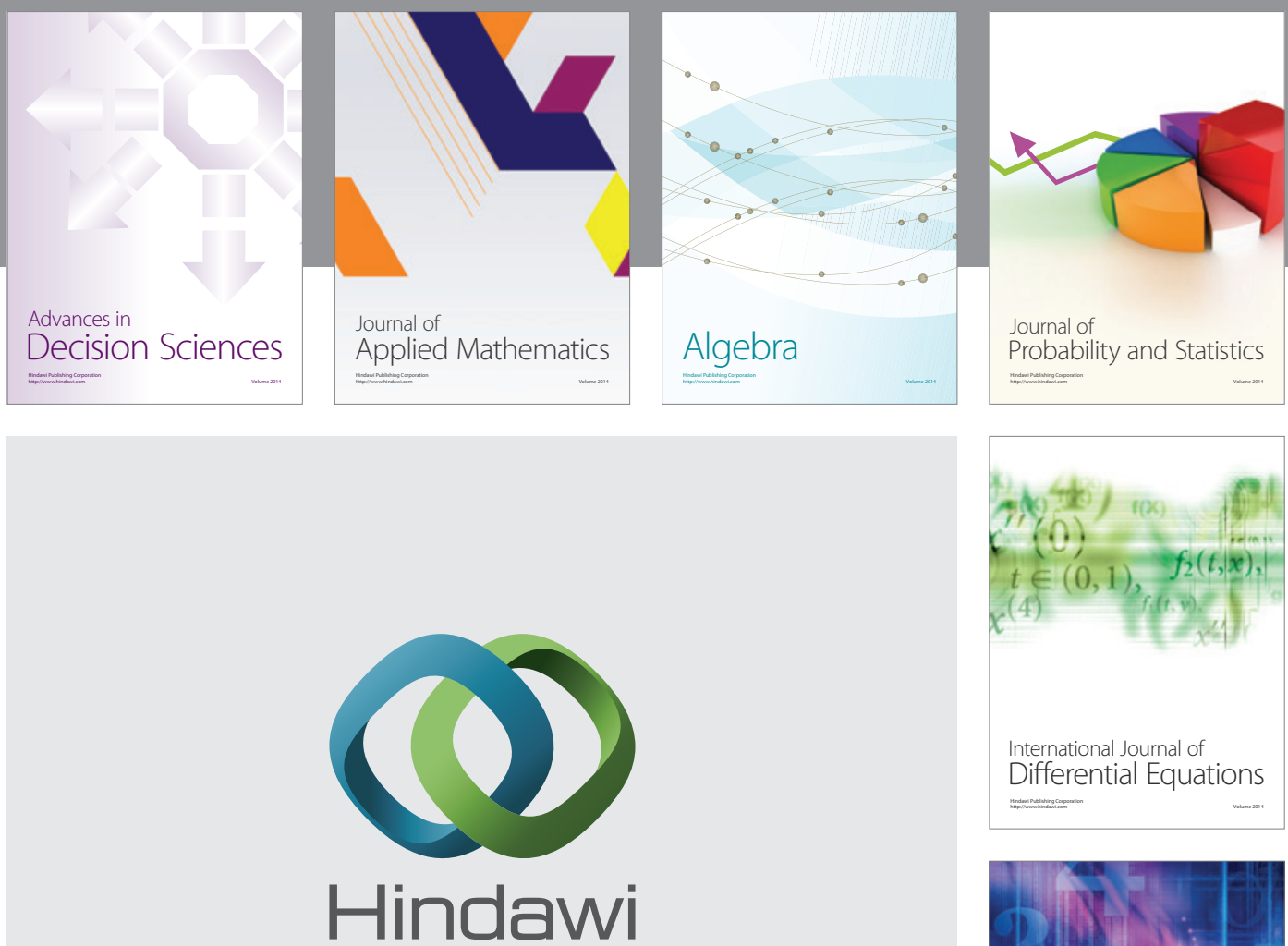

Submit your manuscripts at http://www.hindawi.com
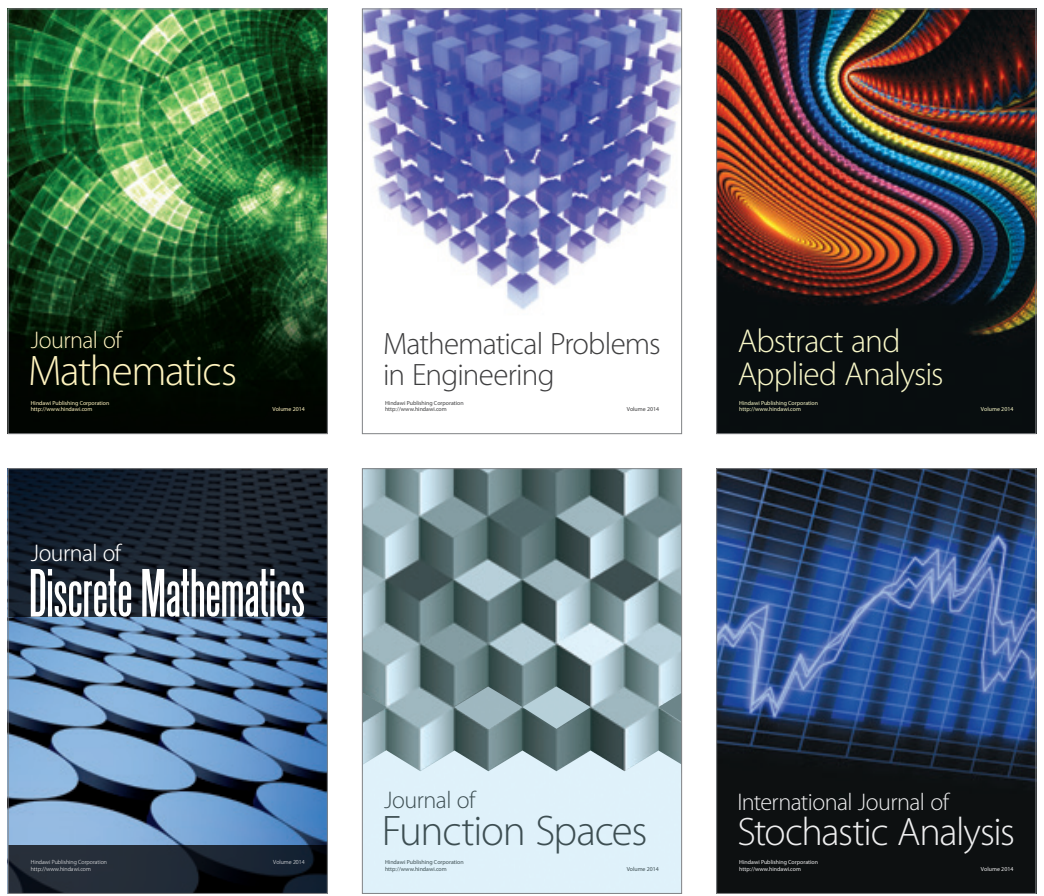

Journal of

Function Spaces

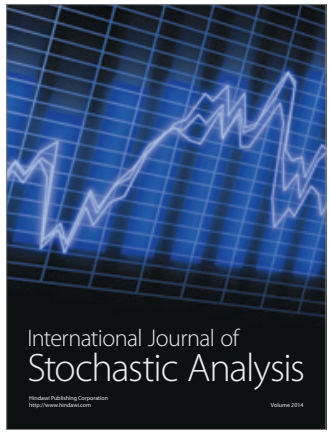

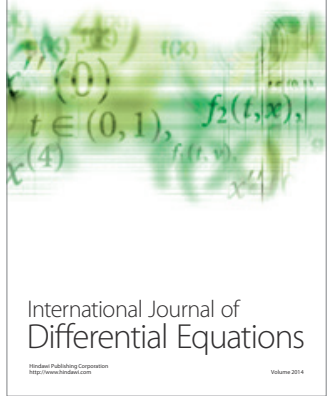
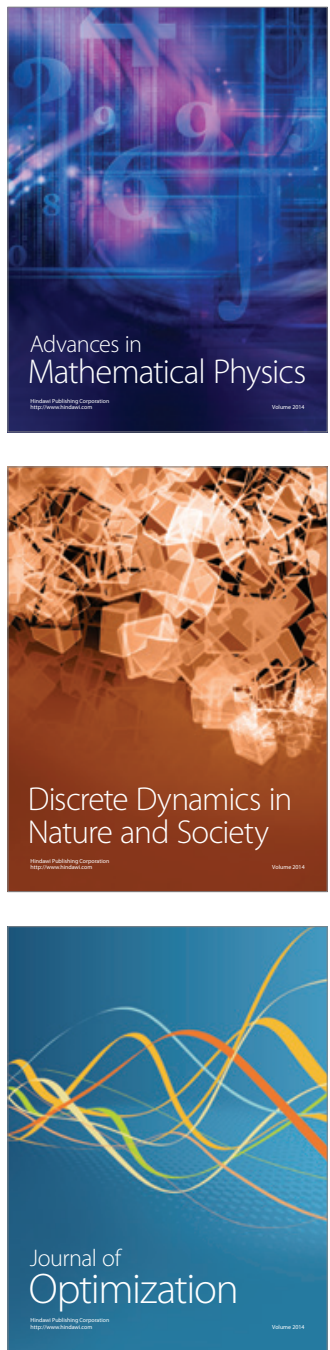